\title{
Development of quantitative methods for detecting climate contributions to boundary shifts in farming-pastoral ecotone of northern China
}

\author{
SHI Wenjiao ${ }^{1,2,3}$, LIU Yiting ${ }^{1,3},{ }^{*}$ SHI Xiaoli ${ }^{4}$
}

1. Key Laboratory of Land Surface Pattern and Simulation, Institute of Geographic Sciences and Natural Resources Research, CAS, Beijing 100101, China;

2. State Key Laboratory of Remote Sensing Science, Beijing Normal University, Beijing 100875, China;

3. College of Resources and Environment, University of Chinese Academy of Sciences, Beijing 100049, China;

4. Key Laboratory of Environmental Evolvement and Ecological Construction of Hebei Province, College of Resources and Environment Sciences, Hebei Normal University, Shijiazhuang 050024, China

\begin{abstract}
The quantitative effect of climate change on fragile regions has been a hot topic in the field of responses to climate change. Previous studies have qualitatively documented the impacts of climate change on boundary shifts in the farming-pastoral ecotone (FPE); however, the quantitative methods for detecting climate contributions remain relatively limited. Based on long-term data of meteorological stations and interpretations of land use since 1970, climate and land use boundaries of the 1970s, 1980s, 1990s and 2000s were delineated. To detect climate contributions to the FPE boundary shifts, we developed two quantitative methods to explore the spatial-temporal pattern of climate and land use boundary at the east-west (or south-north) (FishNet method) and transect directions (Digital Shoreline Analysis System, DSAS method). The results indicated that significant differences were exhibited in climate boundaries, land use boundaries, as well as climate contributions in different regions during different periods. The northwest FPE had smaller variations, while the northeast FPE had greater shifts. In the northwest part of the southeast fringe of the Greater Hinggan Mountains and the Inner Mongolian Plateau, the shifts of climate boundaries were significantly related to the land use boundaries. The climate contributions at an east-west direction ranged from $10.7 \%$ to $44.4 \%$, and those at a south-north direction varied from $4.7 \%$ to $55.9 \%$. The majority of the results from the DSAS were consistent with those from the FishNet. The DSAS method is more accurate and suitable for precise detection at a small scale, whereas the FishNet method is simple to conduct statistical analysis rapidly and directly at a large scale. Our research will be helpful to adapt to climate change, to develop the productive potential, as well as to protect the environment of the FPE in northern China.
\end{abstract}

Keywords: farming-pastoral ecotone (FPE) in northern China; climate change; land use; contribution; quantitative detection

Received: 2017-03-12 Accepted: 2017-04-15

Foundation: National Natural Science Foundation of China, No.41401113, No.41371002; Foundation of Excellent Young Talents of IGSNRR, CAS, No.2016RC201; The Open Fund of State Key Laboratory of Remote Sensing Science, No.OFSLRSS201622; The Key Project of Physical Geography of Hebei Province; China Scholarship Council

Author: Shi Wenjiao, PhD and Associate Professor, specialized in global change and agriculture. E-mail: shiwj@lreis.ac.cn

*Corresponding author: Shi Xiaoli, PhD and Associate Professor, E-mail: hnushixiaoli@163.com 


\section{Introduction}

The farming-pastoral ecotone (FPE), which is a transitional region between agricultural cultivation and pasture (Zhao and $\mathrm{Li}, 2009$ ), is particularly susceptible to climate change and anthropogenic disturbances (Liu and Gao, 2008a). In this region, cropland and grassland are changed frequently over different spatial and temporal scales (Zhao et al., 2002). In China, the FPE are mainly located in the South, Southwest and Northwest regions. As the most extensive spatial extent with fragile environment, the FPE in northern China has attracted the scholars who are interested in climate change and land use change, focusing on boundary fluctuation of the FPE.

From the delineation of the FPE boundary, various qualitative studies indicated that the climate and land use boundaries shifted at opposite directions (Liu and Gao, 2008b; Li and Pan, 2012). Some researchers also found that climate, policy and reclamation together dominated the processes of land use and land cover change, causing the fluctuations of boundaries (Ye and Fang, 2012), especially in Northeast China. A growing number of researchers have quantitatively investigated the influences of climate change and human activities on land use patterns (Shi and Shi, 2015). Models, like the Conversion of Land Use and its Effects Model (CLUE) (Gao and Yi, 2012) and Environment for Geoprocessing Objects Model (Dinamica EGO) (Deng and Zhan, 2004), are often combined with economic models to identify the contributions of natural and anthropogenic impacts on land use change and to project the future pattern in the FPE in northern China (Yang et al., 2014). However, some problems including parameter acquisition, sophisticated operation, and inconsistent validation restricted the application of model analysis. Mathematical statistics such as logistic regression (Gao and Yi, 2012) and pearson correlation ( $\mathrm{Su}$ et al., 2015) can focus on the impacts of a specific climatic factor on land use change in the FPE in northern China. Previous studies focused on the impact analysis at point (Liu and Gao, 2008b; Gao and Liu, 2006) or polygon scales (Ye and Fang, 2013; Shi et al., 2014). However, the methods for detecting quantitative contributions of climate change on boundary shift of the FPE in northern China are still unclear, particularly on line analysis of the boundary (Liu and Gao, 2008a; Liu and Gao, 2008b). Moreover, most of the previous analysis considered the study area as a whole region, which ignored the spatial heterogeneity in the contributions of climate change to the boundary shifts.

Based on the delineation of climate and land use boundaries of the FPE in northern China, we presented two methods to quantitatively measure the contributions of climate change to the boundary fluctuation at $1 \mathrm{~km}$ resolution across different periods and eco-regions, which can detect the impacts of climate change on the boundary fluctuation at the east-west and south-north directions as well as transect directions, respectively. In addition, we also conducted comparisons between the two methods, as well as the analysis of their advantages and limitations.

\section{Data and methods}

\subsection{Study area}

The FPE in northern China, including the southeast fringe of the Greater Hinggan Mountains, the southern fringe of the Inner Mongolian Plateau, and the northern Loess Plateau, as well as a part of the Hexi Corridor, lies between $102^{\circ} 40^{\prime} \mathrm{E}-126^{\circ} 14^{\prime} \mathrm{E}$ and $34^{\circ} 16^{\prime} \mathrm{N}-48^{\circ} 57^{\prime} \mathrm{N}$. It 
covers an area of about $61.4 \times 10^{4} \mathrm{~km}^{2}$, and is located in ten provincial regions including Inner Mongolia, Ningxia, Heilongjiang, Jilin, Liaoning, Hebei, Beijing, Shanxi, Shaanxi and Gansu. In the study area, the annual mean temperature ranges from 0 to $10^{\circ} \mathrm{C}$, and the mean annual precipitation is between $300 \mathrm{~mm}$ and $500 \mathrm{~mm}$. The elevations in most of the study area are over $1000 \mathrm{~m}$. The cropland, grassland and forest are demonstrated staggered distribution. The major land use type is grassland in the northwest FPE, while cropland is predominant in the southeast FPE. The vegetation is typical dry steppe, varying from forest-steppe to steppe and desert steppe. Moreover, due to the rapid increase of economy and population in the southeast FPE during the past 40 years, extensive lands were reclaimed. Consequently, the boundaries were significantly disturbed by human activities in this region. Because different natural and human factors dominated in different regions of the FPE in northern China, four eco-regions are divided to obtain specific results of the climate contributions to the FPE boundary shifts. Based on the division of Huang et al. (2010), we buffered the eco-regions as far as totally covering the whole FPE. The four eco-regions in the study area were created, including the restricted cultivation and water conservation region in the southeast fringe of the Greater Hinggan Mountains (region 1), the agricultural-forestry-pastoral production region in the southeast fringe of the Inner Mongolian Plateau (region 2), the farming-pastoral and soil-water conservation region in northern Loess Plateau (region 3), and the arid desert-oasis cultivation region in the Hexi Corridor (region 4) (Figure 1 and Table 1). Then, the $400 \mathrm{~mm}$ isotype was further used to divide the four eco-regions into the northwest (NW) and southeast (SE) parts (Figure 1 and Table 1).

Table 1 Ecological function regions in the FPE of northern China

\begin{tabular}{lll}
\hline \multicolumn{1}{c}{ Regions } & \multicolumn{1}{c}{ Ecological function regions } & \multicolumn{1}{c}{ Primary ecological functions } \\
\hline Northwest part of region 1 (NW-1) & $\begin{array}{l}\text { The restricted cultivation and water con- } \\
\text { servation region in the southeast fringe of } \\
\text { the Greater Hinggan Mountains } \\
\text { The agricultural-forestry-pastoral and } \\
\text { ecological production region in the } \\
\text { southeast fringe of the Inner Mongolian } \\
\text { Plateau }\end{array}$ & $\begin{array}{l}\text { Agricultural-pastoral production, } \\
\text { forestry-fruit production, and } \\
\text { ecological tourism } \\
\text { Agricultural-pastoral production, } \\
\text { and desertification mitigation }\end{array}$ \\
Southeast part of region 2 (SE-2) & $\begin{array}{l}\text { The farming-pastoral and soil-water con- } \\
\text { servation region in northern Loess Pla- }\end{array}$ & $\begin{array}{l}\text { Soil-water conservation, and } \\
\text { agricultural-forestry production }\end{array}$ \\
teau & The arid desert-oasis cultivation region in \\
the Hexi Corridor & $\begin{array}{l}\text { Desertification mitigation, and } \\
\text { agricultural production }\end{array}$ \\
Noutheast part of region 3 (SE-3) & & \\
\hline
\end{tabular}

\subsection{Data}

\subsubsection{Climate data}

The daily temperature and precipitation data (1970-2010) were from 197 national meteorological stations. ANUSPLIN was employed to obtain raster datasets of climate variables at a $1 \mathrm{~km}$ resolution. The daily temperature dataset was represented by the homogenized daily temperature series for China with the Multiple Analysis of Series for Homogenization (MASH), which can remove systematic bias from time and instruments of observation (Li and Yan, 2009). 


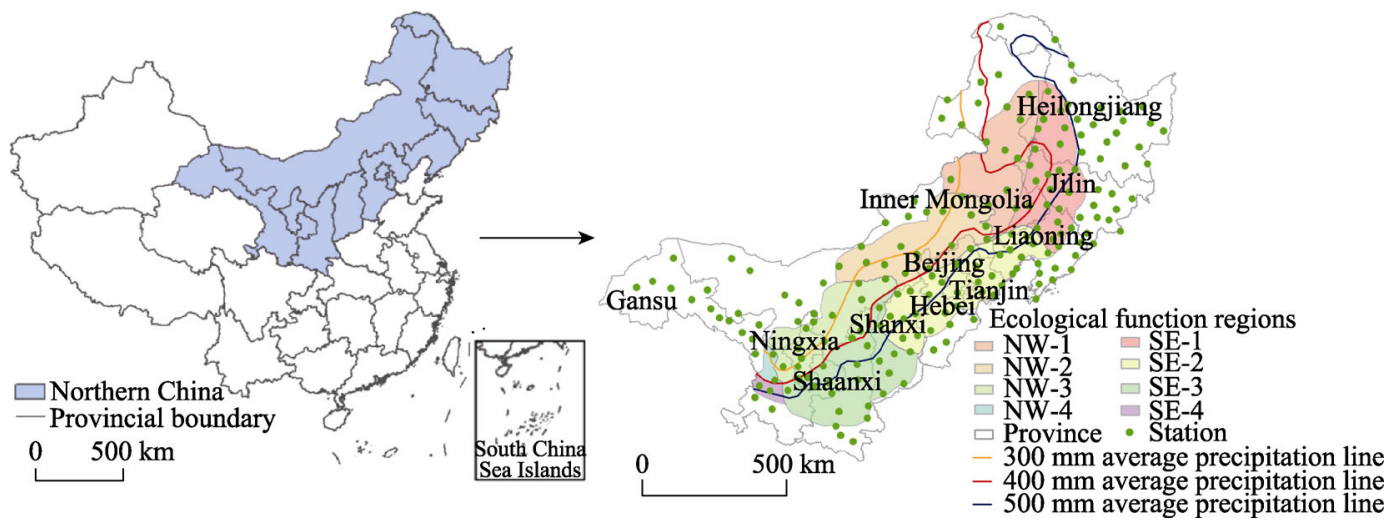

Figure 1 The distributions of ecological function regions and isohyets in the FPE of northern China

\subsubsection{Land use data}

We obtained the time-series dataset of $1 \mathrm{~km}^{2}$ area percentage land use data $(1970 \mathrm{~s}, 1980 \mathrm{~s}$, 1990s and 2000s) from the National Land Cover Dataset (NLCD) (Liu et al., 2003; Liu et al., 2005a; Liu et al., 2010) to investigate the dramatic changes during the past 40 years, which were based on the interpretation of Landsat TM/ETM/MSS, CBERS-1 and CBERS-2. The land use category included cropland, grassland, forest, water body, built-up land and unused land in the NLCD (Liu et al., 2005b; Zhang et al., 2012).

\subsection{Method}

\subsubsection{Delineation of boundaries based on climate and land use data}

Recent works have presented various indices to define the spatial extent of the FPE in northern China. There are two types of indices usually used to delineate the FPE boundary, including climate index and land use index.

For the definition of climate boundary, Zhu et al. (1984) considered that the mean annual precipitation in the FPE varies between 300 and $500 \mathrm{~mm}$. Based on the location of the south fringe of the Inner Mongolian Plateau and the Great Wall, Zhao et al. (2002) selected the mean annual precipitation between 300 and $450 \mathrm{~mm}$, the precipitation variability between $15 \%$ and $30 \%$, and the aridity index between 1 and 2 to delineate the spatial extent of the FPE. Similarly, Liu and Gao (2008b) modified the scope from Zhao et al. (2002) with the precipitation variability (between $15 \%$ and $30 \%$ ) and acidity index (between 0.2 and 0.5 ). Furthermore, they set the $400 \mathrm{~mm}$ isotype as the central line, and used the $300 \mathrm{~mm}$ and 450 $\mathrm{mm}$ isotypes to modify the northwest and southeast boundaries, respectively. Based on the previous research, we defined the climate boundary as follows: (i) the precipitation variability varies from $15 \%$ to $30 \%$ (Liu and Gao, 2008b); (ii) the acidity index varies from 0.2 to 0.5 (Liu and Gao, 2008b); (iii) the $400 \mathrm{~mm}, 300 \mathrm{~mm}$ and $500 \mathrm{~mm}$ isotypes are set as the central line, northwest boundary and southeast boundary, respectively. The isotypes with an interval of $50 \mathrm{~mm}$ from $300 \mathrm{~mm}$ to $500 \mathrm{~mm}$ were used to connect the northwest and southeast boundaries to complete closed boundaries. For a given part of the boundary during different periods, the same isotypes were used to make fair comparison.

For the definition of land use, Ye and Fang (2012) considered that the area-percentage of cropland should be higher than $15 \%$ in the FPE. Wang and Shi (1988) chose the area- 
percentage of cropland (between 15\% and 35\%) and grassland (between 35\% and 75\%) to define the FPE of Inner Mongolia. Actually, the cropland proportion for the whole FPE should be higher than those in Inner Mongolia. Wu and Guo (1994) presented that the proportion for cropland, grassland and forest should be 1:0.5:1.5 in the FPE. Therefore, we defined land use boundary as follows: (i) both the area-percentages of cropland and grassland should be greater than $15 \%$ in each $1 \mathrm{~km} \times 1 \mathrm{~km}$ grid; and (ii) the spatial extent should be contiguous distribution and also be contained in the four eco-regions.

\subsubsection{Detection of the boundary shifts at the east-west and south-north directions}

This method could distinguish the boundary shift at the east-west and south-north directions with the FishNet operation in ArcGIS. The $1 \mathrm{~km} \times 1 \mathrm{~km}$ fishnet was created to entirely cover the largest scope of the FPE over the four periods, the shift distances and directions for both of the climate and land use boundaries on each fishnet line were computed at two directions in each period. When a fishnet line crossed the boundaries during former period and latter period from east to west (or from south to north), the direction was coded as 1 . The direction was set as -1 if it crossed boundaries from the opposite directions. The direction was coded as 0 for the boundaries of the same period crossed by a fishnet line. The distance signed with direction could be computed by the equation (1),

$$
D_{f p-l p}=L_{f p-l p} \times \text { Direction }
$$

where $f p$ is the former period, $l p$ is the latter one; $L_{f p-l p}$ represents the length of fishnet line; Direction represents the shift direction of boundaries; $D_{f p-l p}$ represents the distance with direction sign. The $L_{f p-l p}$ was used to compute the maximum, minimum, median, as well as the mean of the shift distance for climate and land use boundaries, and the $D_{f p-l p}$ was used to examine the shift ranges and correlations between climate and land use boundaries.

\subsubsection{Detection of the boundary shifts at the transect direction}

A baseline was created according to the buffer of the innermost boundary, and then transects were casted perpendicularly to the baseline with an internal of $1 \mathrm{~km}$ and intersected the boundaries during different periods using the Digital Shoreline Analysis System (DSAS method) (Wang et al., 2014; Jayson et al., 2013). The distance $\left(D_{p}\right)$ from the baseline to the intersect point of each boundary was computed. The shift distance and direction of climate and land use boundaries could be computed according to the equation (2),

$$
D_{f p-l p}=D_{l p}-D_{f p}
$$

where $D_{f p}$ represents the distance between the boundary in the former period and the baseline, $D_{l p}$ represents the distance between the latter boundary and the baseline, $D_{f p-l p}$ represents the distance between the former and latter boundaries marked with direction. The absolute values of $D_{f p-l p}$ can be used to compute the maximum, minimum, median, as well as the mean of the shift distance for climate and land use boundaries. The $D_{f p-l p}$ was subsequently used to examine the shift ranges and correlations between climate and land use boundaries.

\subsubsection{Calculation of the climate contributions to boundary shifts}

The shift distances $\left(D_{f p-l p}\right)$ of the climate and land use boundaries at the east-west, south-north and transect directions in each eco-region were calculated using both methods of the FishNet and DSAS. The correlation, coefficient $\left(r^{2}\right)$ and significance $(p)$ were calculated according to the shift distances of the climate and land use boundaries. To explore how 
much of land use boundary shifts can be explained by climate change, we selected the datasets with significantly positive correlations $(p<0.05, r>0)$ between climate and land use boundaries shifts, and selected the coefficient $\left(r^{2}\right)$ to demonstrate the contributions of climate change to the FPE boundary shifts.

\section{Results and discussion}

We used the FishNet and DSAS methods to detect the spatial-temporal pattern changes of the FPE boundaries based on the data of climate and land use. Quantitative detection of the climate contributions to the FPE boundary shifts was then conducted using these two methods.

\subsection{Spatial-temporal pattern changes of the FPE boundaries detected by the FishNet method}

\subsubsection{Climate boundary}

The greatest change of climate boundary shifted eastward in SE-2 region from the 1990s to the 2000s with $278.54 \mathrm{~km}$ at the east-west direction (Figures 2a, 2c and 2e). Extensive shifts existed in region 1, NW-2 and SE-3 regions. For example, during the 1990s-2000s, the FPE boundaries in NW-1 and NW-2 regions significantly moved eastward with the median shift distances of $91.88 \mathrm{~km}$ and $78.76 \mathrm{~km}$, while it moved westward with $72.02 \mathrm{~km}$ in the SE-3 region. In contrast, the FPE boundaries in region 4 varied more slightly with the median distances less than $10 \mathrm{~km}$.

The largest change of climate boundary shifted southward with $271.25 \mathrm{~km}$ at the south-north direction in NW-1 region from the 1970s to the 1980s (Figures 2b, 2d and 2f). The climate boundaries in the southeast (SE) part experienced greater shifts than those in the northwest (NW) part. More extensive changes of the FPE boundaries can be detected in region 1, SE-2 and SE-3 regions, while the boundaries in NW-4 region continuously moved northward with slight changes. As an increase of precipitation in Northeast China during the 1980s, the FPE boundaries in region 1, NW-3 and SE-2 regions shifted southward during the 1970s-1980s. The median shift distances of the NW and the SE parts in region 1 were 97.28 $\mathrm{km}$ and $84.93 \mathrm{~km}$, respectively. After that period, the climate boundaries gradually moved northward with a decrease of precipitation.

\subsubsection{Land use boundary}

The land use boundary shifted eastward with $140.39 \mathrm{~km}$ at the east-west direction in SE-2 region during the 1970s-1980s, which was the most extensive shift in the whole FPE area during the study periods (Figures 2a, 2c and 2e). Similarly, extensive shifts of the land use boundaries were found in region 1 and region 2. During the 1980s-1990s, the boundary in $\mathrm{NW}-1$ region mainly experienced westward moving with a median distance of $32.34 \mathrm{~km}$, while boundaries moved slightly in region 4, with the median distances lower than $7 \mathrm{~km}$. However, for the whole FPE boundaries, there were no significant differences between the SE and NW parts due to the boundary fluctuations.

At the south-north direction, the largest change of land use boundary shifting northward with $149.82 \mathrm{~km}$ was observed in NW-2 region during the 1970s-1980s (Figures 2b, 2d and $2 \mathrm{f})$. Boundaries shifts in region 1 were more extensive than those in the others. A significant northward moving was detected in SE-1 region during the 1990 s-2000s, with a median dis- 
tance of $46.07 \mathrm{~km}$. The boundaries in regions 2, 3 and 4 experienced minor shifts, of which median distances were lower than $10 \mathrm{~km}$ with the exception of those in NW-4 region with $10.82 \mathrm{~km}$ during the $1980 \mathrm{~s}-1990 \mathrm{~s}$.
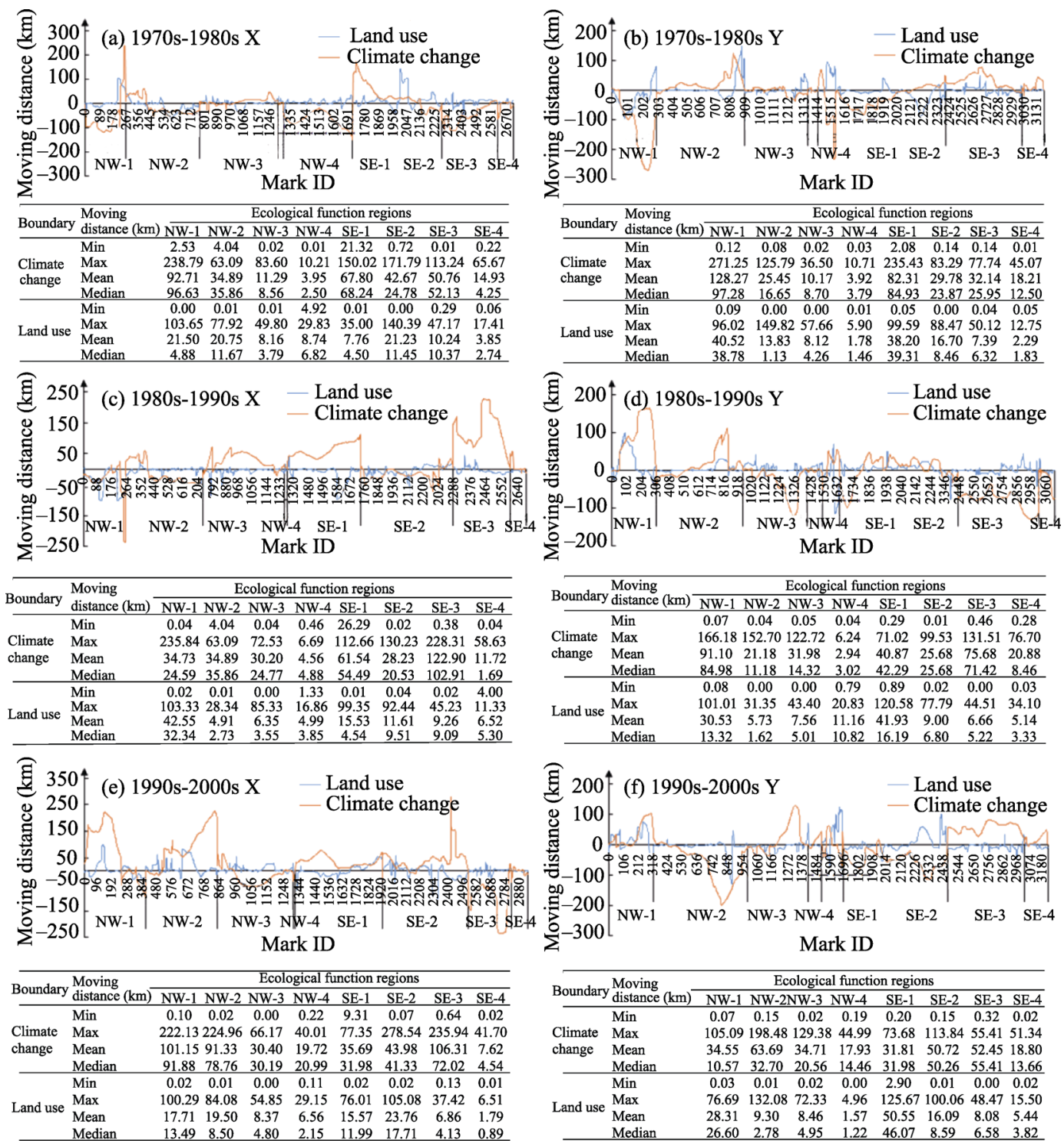

Figure 2 Changes of climate and land use boundaries detected by the FishNet method

\subsection{Spatial-temporal pattern changes of the FPE boundaries detected by the DSAS method}

\subsubsection{Climate boundary}

The climate boundaries experienced more extensive shifts than land use boundaries. The largest change of climate boundary was observed in SE-1 region during the 1970s-1980s, which shifted southward with $299.09 \mathrm{~km}$ (Figures 3a, 3c and 3e). Similarly, we found considerable shifts in regions 1 and 2 detected by the FishNet. The most extensive shift was 
detected during the 1970s-1980s in SE-1 region, which shifted westward with the median shift distance of $103.75 \mathrm{~km}$. On the contrary, boundaries in region 4 experienced slight fluctuations, most of medians of shift distances were lower than $5 \mathrm{~km}$, except for those in the NW-4 region during the $1990 \mathrm{~s}-2000 \mathrm{~s}(16.71 \mathrm{~km})$. For boundary fluctuations of the whole FPE, the greater changes were observed in the SE part than those in the NW part.

\subsubsection{Land use boundary}

The largest change of land use boundary was observed with a shift of $217.79 \mathrm{~km}$ moving northeastward in SE-1 region from the 1990s to the 2000s (Figures 3b, 3d and 3f). The highest median was found in NW-1 region, which was $28.89 \mathrm{~km}$ shifted northwestward during the 1980s-1990s. Similar to the results from the FishNet, there were no significant shifts in regions 2, 3 and 4. The medians of shift distances in region 4 were lower than $10 \mathrm{~km}$ except for that in the NW-4 region during the 1980s-1990s $(12.39 \mathrm{~km})$.
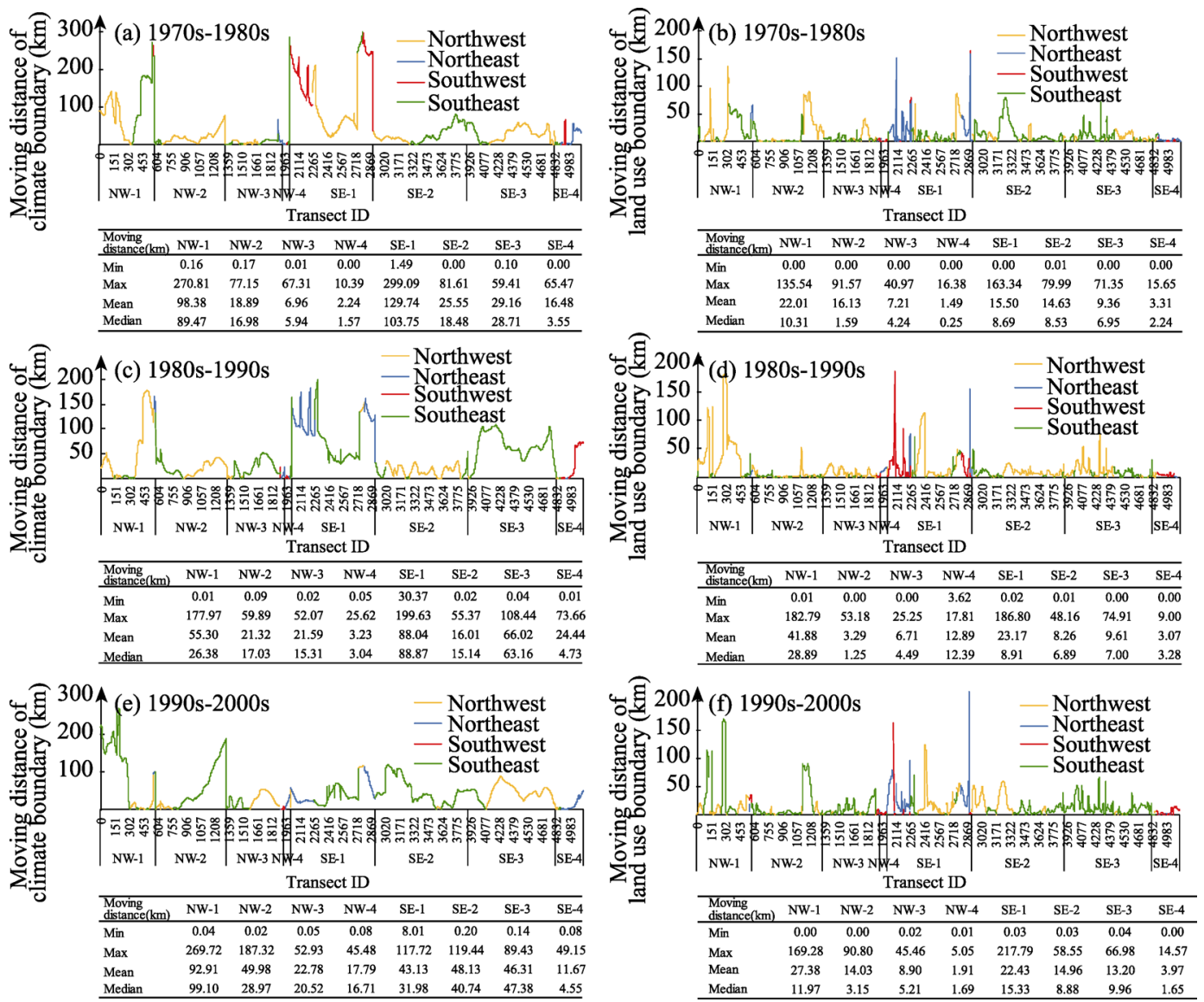

Figure 3 Changes of climate and land use boundaries detected by the DSAS method

\subsection{Quantitative detection of the climate contributions to the FPE boundary shifts using the FishNet method}

The climate contributions to the FPE boundary shifts significantly varied among regions and periods detected by the FishNet method (Table 2). 
Table 2 The quantitative detection of climate change effects on the boundary fluctuation in the FPE of northern China

\begin{tabular}{|c|c|c|c|c|c|c|c|}
\hline \multirow{2}{*}{ Regions } & \multirow{2}{*}{ Periods } & \multicolumn{2}{|c|}{$\mathrm{X}$ direction } & \multicolumn{2}{|c|}{ Y direction } & \multicolumn{2}{|c|}{ Transect direction } \\
\hline & & Sample numbers & $r^{2}$ & Sample numbers & $r^{2}$ & Sample numbers & $r^{2}$ \\
\hline \multirow{3}{*}{ NW-1 } & $1970 \mathrm{~s}-1980 \mathrm{~s}$ & 261 & 0.444 & - & - & 580 & 0.027 \\
\hline & $1980 s-1990 s$ & 255 & 0.107 & - & - & - & - \\
\hline & $1990 s-2000 s$ & 411 & 0.202 & 331 & 0.047 & 580 & 0.011 \\
\hline \multirow{3}{*}{ NW-2 } & $1970 \mathrm{~s}-1980 \mathrm{~s}$ & 473 & 0.209 & 655 & 0.088 & 755 & 0.168 \\
\hline & $1980 \mathrm{~s}-1990 \mathrm{~s}$ & - & - & 726 & 0.559 & - & - \\
\hline & $1990 \mathrm{~s}-2000 \mathrm{~s}$ & - & - & 697 & 0.104 & 755 & 0.043 \\
\hline \multirow{3}{*}{ NW-3 } & $1970 \mathrm{~s}-1980 \mathrm{~s}$ & - & - & - & - & - & - \\
\hline & $1980 \mathrm{~s}-1990 \mathrm{~s}$ & - & - & 457 & 0.082 & 612 & 0.043 \\
\hline & $1990 \mathrm{~s}-2000 \mathrm{~s}$ & - & - & - & - & - & - \\
\hline \multirow{3}{*}{ NW-4 } & $1970 \mathrm{~s}-1980 \mathrm{~s}$ & - & - & 77 & 0.052 & 73 & 0.153 \\
\hline & $1980 s-1990 s$ & - & - & - & - & - & - \\
\hline & $1990 \mathrm{~s}-2000 \mathrm{~s}$ & - & - & - & - & - & - \\
\hline \multirow{3}{*}{ SE-1 } & $1970 \mathrm{~s}-1980 \mathrm{~s}$ & - & - & - & - & - & - \\
\hline & $1980 \mathrm{~s}-1990 \mathrm{~s}$ & - & - & - & - & - & - \\
\hline & $1990 \mathrm{~s}-2000 \mathrm{~s}$ & - & - & - & - & 891 & 0.015 \\
\hline \multirow{3}{*}{ SE-2 } & $1970 \mathrm{~s}-1980 \mathrm{~s}$ & - & - & - & - & - & - \\
\hline & $1980 \mathrm{~s}-1990 \mathrm{~s}$ & 567 & 0.040 & - & - & 985 & 0.032 \\
\hline & $1990 \mathrm{~s}-2000 \mathrm{~s}$ & - & - & - & - & - & - \\
\hline \multirow{3}{*}{ SE-3 } & $1970 \mathrm{~s}-1980 \mathrm{~s}$ & 357 & 0.201 & 568 & 0.227 & 932 & 0.099 \\
\hline & $1980 \mathrm{~s}-1990 \mathrm{~s}$ & - & - & - & - & - & - \\
\hline & $1990 \mathrm{~s}-2000 \mathrm{~s}$ & - & - & - & - & 932 & 0.019 \\
\hline \multirow{3}{*}{ SE-4 } & $1970 s-1980 s$ & - & - & - & - & - & - \\
\hline & $1980 \mathrm{~s}-1990 \mathrm{~s}$ & - & - & - & - & - & - \\
\hline & $1990 \mathrm{~s}-2000 \mathrm{~s}$ & - & - & - & - & - & - \\
\hline
\end{tabular}

In different eco-regions of the FPE, climate had pronounced impacts on the FPE boundary fluctuations in NW-1 region, with the contributions from $10.7 \%$ to $44.4 \%$ at the east-west direction, while the climate contribution at the south-north direction was only $4.7 \%$ during the 1990s-2000s. In region 2, climate change significantly influenced boundary fluctuations in the NW part with climate contributions from $8.8 \%$ to $55.9 \%$ at the south-north direction in the three periods. However, the boundary during the 1970s-1980s was only largely driven by climate change with a contribution of $20.9 \%$ at the east-west direction. In addition, climate change hardly impacted the boundaries in the SE part; only $4.0 \%$ of the climate contribution was detected at the east-west direction during the $1980 \mathrm{~s}-1990 \mathrm{~s}$. In region 3 , the climate contribution was $8.2 \%$ at the south-north direction during the $1980 \mathrm{~s}-1990 \mathrm{~s}$ in the NW part. On the contrary, remarkable climate impacts with contributions of $20.1 \%$ and $22.7 \%$ were observed in both directions during the $1970 \mathrm{~s}-1980 \mathrm{~s}$. The climate contributions were also very low in region 4 , in which climate change only significantly drove the boundary shift with a contribution of $5.2 \%$ in the NW part at the south-north direction during the 1970s-1980s.

During different periods, climate change had different contributions to the FPE boundary shifts. The climate contributions in NW-1, NW-2 and SE-3 regions varied from $20.1 \%$ to $44.4 \%$ at the east-west direction in the 1970s-1980s. Meanwhile, the contributions of cli- 
mate change in NW-2, NW-4 and SE-3 regions varied from $5.2 \%$ to $22.7 \%$ at the south-north direction. During the 1980s-1990s, the contributions of climate change in NW-1 and SE-2 regions were between $4.0 \%$ and $10.7 \%$ at the east-west direction, while the contributions of climate change in NW-2 and NW-3 regions ranged from $8.2 \%$ to $55.9 \%$ at the south-north direction. During the 1990s-2000s, climate significantly influenced the NW-1 and NW-2 regions. The contribution of climate change in NW-1 region got up to $20.2 \%$ at the east-west direction during this period, and the contributions in NW-1 and NW-2 regions at the south-north direction were $4.7 \%$ and $10.4 \%$, respectively.

Detected by the FishNet method, the greatest climate contribution was $44.4 \%$ in NW-1 region at the east-west direction during the 1970s-1980s. In addition, it was $55.9 \%$ at the south-north direction in NW-2 region during the 1980s-1990s. Higher contributions of climate change were concentrated in NW-1 and NW-2 regions, which were between $10.7 \%$ and $44.4 \%$ at the east-west direction and ranging from $4.7 \%$ to $55.9 \%$ at the south-north direction. The results indicated that the FPE boundary changes were largely driven by climate in Northeast China.

\subsection{Quantitative detection of the climate contributions to the FPE boundary shifts using the DSAS method}

The climate contributions in different regions and periods detected by the DSAS method were similar to those detected by the FishNet method (Table 2).

The FPE boundaries in regions 1 and 2 were considerably shaped by climate change. The contributions during the $1970 \mathrm{~s}-1980 \mathrm{~s}$ and $1990 \mathrm{~s}-2000 \mathrm{~s}$ in $\mathrm{NW}-1$ region were $2.7 \%$ and $1.1 \%$, respectively. Furthermore, the climate contribution during the $1990 \mathrm{~s}-2000 \mathrm{~s}$ in SE-1 region was $1.5 \%$. For NW-2 region, the climate contributions to land use boundaries were $16.8 \%$ and $4.3 \%$ during the $1970 \mathrm{~s}-1980 \mathrm{~s}$ and $1990 \mathrm{~s}-2000 \mathrm{~s}$, respectively. It was $3.2 \%$ in SE-2 region during 1980s-1990s. Significant impacts of climate on the FPE boundaries were limited in regions 3 and 4. The contributions in SE-3 region were higher than those in NW-3 region. The contributions were $9.9 \%$ and $1.9 \%$ in SE-3 region during the $1970 \mathrm{~s}-1980 \mathrm{~s}$ and 1990s-2000s, respectively. The climate contribution in NW-3 region was $4.3 \%$ during the 1980s-1990s. However, the climate contribution in the NW-4 region was $15.3 \%$ during the 1970s-1980s.

Climate had pronounced influences on land use boundaries during the 1970s to the 1980s. The climate contributions in the NW part ranged from $2.7 \%$ to $16.8 \%$, which were slightly higher than those in the SE part. During the 1980s-1990s, the climate contributions exhibited lower values than those in the $1970 \mathrm{~s}-1980 \mathrm{~s}$, which varied from $3.2 \%$ to $4.3 \%$. From the 1990 s to the 2000 s, land use boundaries in region 1, NW-2 and SE-3 regions were all influenced by climate, in which the climate contributions were between $1.1 \%$ and $4.3 \%$.

Using the DSAS method, the largest contribution with $16.8 \%$ was observed in NW-2 region during the 1970s-1980s. Higher contributions existed in NW-1, NW-2, NW-4 and SE-3 regions during the 1970 s-1980s.

\subsection{Discussion}

\subsubsection{Compared with the results from previous research}

Several studies showed that climate warming had caused cropland expansion in Northeast China since the 1980s (Ye et al., 2012; Liu et al., 2009; Wang et al., 2009), which fitted well 
with the obvious climate contributions in NW-1 and NW-2 regions in our study. Liu et al. (2011) found that the FPE boundary in the SE part of Northeast China was influenced by human activities from 1986 to 2000, and cropland in this region showed a predominant expansion to the west, which was in accordance with the results of insignificant climate impacts on the FPE shifts in SE-1 region during the former two periods. This conclusion was similar to the results in SE-1 region in our study. Furthermore, the implement of the Green for Grain Project and Grazing Prohibition policy affected the boundary shifts in the northwest FPE since 1999, which was in accordance with the insignificant impacts of climate on the FPE boundaries in NW-3 and NW-4 regions during the 1990s-2000s (Lu et al., 2013). Zuo et al. (2014) showed that the land use in western China has been dominated by human activities and policies since the 1980s. This result was also consistent with the insignificant contributions of climate in region 4 since the 1980s, which was located in the northwest of China. Thus, anthropogenic disturbance was also one of the primary drivers of boundary shifts in the FPE of northern China (Yang et al., 2014; Liu et al., 2014).

\subsubsection{Comparison of the two methods of FishNet and DSAS}

Most of the spatial-temporal pattern of boundary shifts detected by the methods of FishNet and DSAS had good consistence with each other. The changes of climate boundaries were at larger extent than those of land use boundaries using both methods. The greatest shifts were detected in region 1 and the smallest shifts were shown in region 4 over the three periods. The mean values and medians of shift distances in most of regions were close to the minimum, suggesting smaller changes of boundaries in these regions. The shift directions of the boundaries based on climate and land use were similar in most of the regions. For example, the shift directions of climate boundaries in regions 2 and 3, as well as land use boundaries in SE-2 and SE-3 regions were coincident detected by the methods of FishNet and DSAS. However, some inconsistencies also existed in some regions. Compared with the FishNet method, the DSAS method is closer to the facts of the detection of directions and distances for the FPE shifts.

Using the two methods, climate contributions to the FPE boundary shifts varied among regions during the study periods, and the results detected by the FishNet and DSAS methods were approximately coincident. For example, during the 1970s-1980s, the climate contributions detected by the FishNet in NW-2 region at the east-west and south-north directions were $20.9 \%$ and $8.8 \%$, respectively, and the contribution was $16.8 \%$ from the DSAS method. Another example was in SE-3 region during the 1970s-1980s. The contributions were $20.1 \%$ and $22.7 \%$ at the east-west and south-north directions detected by the FishNet method, whereas the result from DSAS was 9.9\%. However, differences were also investigated from the two methods. For instance, during the 1980s-1990s, the climate contribution at the south-north direction was $55.9 \%$ in NW-2 region, but the correlation of the shifts based on climate and land use boundaries detected from the DSAS method was not significant in this region.

Overall, the FishNet method distinctly demonstrates the climate impacts on the FPE boundary at the east-west and south-north directions, which is simple but not as accurate as the DSAS method. Therefore, this method can be employed to directly analyze the boundary changes at large scales, if it is not necessary to achieve strict requirement of accuracy. On the contrary, the DSAS method can precisely show the climate contributions to boundary shifts at the transect direction, but it is noted that the results are easily affected by the base- 
line. This method is especially suitable to detect the boundary changes at a small scale. Both of the two methods are primarily influenced by extreme values, and the uncertainties are relatively large in the junctures of eco-regions.

\section{Conclusions}

The FPE boundary fluctuations were not only driven by climate change, but also affected by human activities in northern China. We presented the FishNet method and DSAS method to quantitatively investigate the climate contributions to the boundary shifts at the scale of 1 $\mathrm{km}$ resolution. The results will be helpful for understanding the climate change impacts on fragile regions, and also for determining adaptation measures that should be adopted.

Due to the large area of the FPE in northern China and complicated natural environment of different eco-regions, the boundary shifts have great differences among regions during different periods. We detected these differences in the shifts of climate and land use boundaries using the methods of FishNet and DSAS. The greatest fluctuations were detected in region 1, whereas region 4 had the smallest shifts. Good spatial coupling relationships between the shifts of climate and land use boundaries were detected in the NW-1 and NW-2 regions. Climate contributions of these regions varied from $10.7 \%$ to $44.4 \%$ at the east-west direction, as well as from $4.7 \%$ to $55.9 \%$ at the south-north direction detected by the FishNet method. The climate contributions in these regions were between $1.1 \%$ and $16.8 \%$ detected by the DSAS method. During the study periods, relationship between the shifts of climate boundaries and land use boundaries was not significant in region 4, suggesting that climate change was not the main driving force of the FPE boundary shifts.

Therefore, the conclusions of the detection from the FishNet method and DSAS method had good consistence. The DSAS method is more accurate than the FishNet method, which is a proper one to detect precise changes at a smaller scale. However, the FishNet method is simple to operate, and is suitable to conduct statistical analysis rapidly and directly at a larger scale.

\section{References}

Deng X Z, Zhan J Y, 2004. Scale-effect analysis of LUCC driving forces in the farming-pasturing interlocked area in northern China. Geography and Geo-Information Science, 20(3): 64-68. (in Chinese)

Gao Z Q, Liu J Y, 2006. The LUCC responses to climate changes in China from 1980 to 2000. Acta Geographica Sinica, 61(8): 865-872. (in Chinese)

Gao Z Q, Yi W, 2012. Land use change in China and analysis of its driving forces using CLUE-S and Dinamica EGO model. Transactions of the Chinese Society of Agricultural Engineering, 28(16): 208-216. (in Chinese)

Huang Q, Xin X P, Zhang H B, 2010. Ecosystem-service-based regionalization of the grassland and agro-pastoral transition zone in northern China. Acta Ecologica Sinica, 30(2): 350-356. (in Chinese)

Jayson Q P, Appeaning A K, Kufogbe S, 2013. Medium resolution satellite imagery as a tool for monitoring shoreline change: Case study of the Eastern coast of Ghana. Journal of Coastal Research, 65(sp1): 511-516.

Li Q Y, Pan X B, 2012. The impact of climate change on boundary shift of farming pasture ecotone in northern China. Journal of Arid Land Resources and Environment, 26(10): 1-6. (in Chinese)

Li Z, Yan Z W, 2009. Homogenized daily mean/maximum/minimum temperature series for China from 1960-2008. Atmospheric and Oceanic Science Letters, 2(4): 236-242.

Liu D W, Wang Z M, Song K S et al., 2009. Land use/cover changes and environmental consequences in Songnen Plain, Northeast China. Chinese Geographical Science, 19(4): 299-305.

Liu J H, Gao J X, 2008a. Changes of land use and landscape pattern in the boundary change areas in farming-pastoral ecotone of northern China. Transactions of the Chinese Society of Agricultural Engineering, 
24(11): 76-82. (in Chinese)

Liu J H, Gao J X, 2008b. Spatial changes of boundary based on land use and climate change in the farming-pastoral ecotone of northern China. China Environmental Science, 28(3): 203-209. (in Chinese)

Liu J H, Gao J X, Lv S et al., 2011. Shifting farming-pastoral ecotone in China under climate and land use changes. Journal of Arid Environments, 75(3): 298-308.

Liu J Y, Kuang W H, Zhang Z X et al., 2014. Spatiotemporal characteristics, patterns, and causes of land-use changes in China since the late 1980s. Journal of Geographical Sciences, 24(2): 195-210.

Liu J Y, Liu M L, Tian H Q et al., 2005. Spatial and temporal patterns of China's cropland during 1990-2000: An analysis based on Landsat TM data. Remote Sensing of Environment, 98(4): 442-456.

Liu J Y, Liu M L, Zhuang D F et al., 2003. Study on spatial pattern of land-use change in China during 1995-2000. Science in China. Series D: Earth Sciences, 46(4): 373-384.

Liu J Y, Zhang Z X, Xu X L et al., 2010. Spatial patterns and driving forces of land use change in China during the early 21st century. Journal of Geographical Sciences, 20(4): 483-494.

Liu J Y, Zhang Z X, Zhuang D F et al., 2005. The Study on the Spatial-temporal Information of Land Use Changes in China with Remote Sensing in the 1990s. Beijing: Science Press. (in Chinese)

Lu W, Jia G S, 2013. Fluctuation of farming-pastoral ecotone in association with changing East Asia monsoon climate. Climatic Change, 119(3): 747-760.

Shi W J, Tao F L, Liu J Y et al., 2014. Has climate change driven spatio-temporal changes of cropland in northern China since the 1970s?. Climatic Change, 124(1/2): 163-177.

Shi X L, Shi W J, 2015. Identifying contributions of climate change and human activities to cropland spatial-temporal changes: A review. Acta Geographica Sinica, 70(9): 1463-1476. (in Chinese)

Su W, Liu X X, Luo Q et al., 2015. Responses of vegetation to change of meteorological factors in agricultural-pastoral area of northern China. Transactions of the Chinese Society for Agricultural Machinery, 46(11): 352-359. (in Chinese)

Wang J A, Shi P J, 1988. The utilization of land resources and regional development strategies of farming-pastoral zone in Inner Mongolia. Areal Research and Development, 7(1): 24-28. (in Chinese)

Wang Y D, Hou X Y, Jia M M et al., 2014. Remote detection of shoreline changes in eastern bank of Laizhou Bay, North China. Journal of The Indian Society of Remote Sensing, 42(3): 621-631.

Wang Z M, Liu Z M, Song K S et al., 2009. Land use changes in Northeast China driven by human activities and climatic variation. Chinese Geographical Science, 19(3): 225-230.

Wu C J, Guo H C, 1994. The Land Use of China. Beijing: Science Press. (in Chinese)

Yang Y Y, Zhang S W, Wang D Y et al., 2014. Spatiotemporal changes of farming-pastoral ecotone in northern China, 1954-2005: A case study in Zhenlai County, Jilin Province. Sustainability, 7(1): 1-22.

Ye Y, Fang X Q, 2012. Expansion of cropland area and formation of the eastern farming-pastoral ecotone in northern China during the twentieth century. Regional Environmental Change, 12(4): 923-934.

Ye Y, Fang X Q, 2013. Boundary shift of potential suitable agricultural area in farming-grazing transitional zone in northeastern China under background of climate change during 20th century. Chinese Geographical Science, 23(6): 655-665.

Ye Y, Fang X Q, Khan M A U, 2012. Migration and reclamation in Northeast China in response to climatic disasters in North China over the past 300 years. Regional Environmental Change, 12(1): 193-206.

Zhang Z X, Zhao X L, Wang X, 2012. Remote sensing monitoring of land use in China. Beijing: Star Map Press. (in Chinese)

Zhao H L, Zhao X Y, Zhang T H et al., 2002. Boundary line on agro-pasture zigzag zone in North China and its problems on eco-environment. Advance in Earth Sciences, 17(5): 739-747. (in Chinese)

Zhao J, Li X, 2009. Research progress on the farming-pastoral ecotone in China. Pratacultural Science, 26(1): 94-99. (in Chinese)

Zhu Z D, Liu S, Yang Y L, 1984. The possibilities and realities on re-management of desertified lands in the inter-distributing areas for argicultural and graziery practices in northern China. Scientia Geographica Sinica, 4(3): 197-206. (in Chinese)

Zuo L J, Zhang Z X, Zhao X L et al., 2014. Multitemporal analysis of cropland transition in a climate-sensitive area: A case study of the arid and semiarid region of northwest China. Regional Environmental Change, 14(1): 75-89. 